\title{
The Power Counting Theorem for Minkowski Metric *
}

Wolfhart ZimmermanN

Courant Institute of Mathematical Sciences

and

Physics Department, New York University, New York, N. Y.

Received June 11, 1968 metric.

Abstract. Dyson's power counting theorem is proved for the case of Minkowski

\section{Introduction}

A major complication in conventional renormalization theory has been the fact that even in renormalized form Feynman integrals are only conditionally convergent [1]. In this paper a simple method of circumventing this difficulty is discussed. We propose to write the Feynman propagator in the form

$$
\frac{1}{l_{0}^{2}-\boldsymbol{l}^{2}-\mu^{2}+i \varepsilon\left(\boldsymbol{l}^{2}+\mu^{2}\right)} .
$$

With this convention it is easy to see that the Feynman integrals are absolutely convergent for $\varepsilon>0$ provided the hypothesis of the power counting theorem applies (Section 2). Of course, the integrals are not relativistically covariant as long as $\varepsilon>0$. But it will be shown in section 3 that the limit exists and defines a covariant distribution.

\section{Power Counting Theorem for Minkowski Metric}

We consider Feynman integrals of the form

where

$$
I(q \mu \varepsilon)=\int d k \frac{P(k q)}{\prod_{j=1}^{n} f_{j}\left(k q, \mu_{j} \varepsilon\right)}
$$

$$
\begin{aligned}
q & =\left(q_{1} \ldots q_{r}\right), \quad k=\left(k_{1} \ldots k_{m}\right), \\
d k & =d k_{1} \ldots d k_{m}, \\
\mu & =\left(\mu_{1} \ldots \mu_{n}\right), \quad \mu_{j} \geqq 0,
\end{aligned}
$$

with $q_{j}, k_{j}$ denoting Minkowski four vectors. The functions $f_{j}$ denote Feynman denominators in the modified form

$$
f_{j}\left(k q_{j} \mu_{j} \varepsilon\right)=l_{j 0}^{2}-\boldsymbol{l}_{j}^{2}-\mu_{j}^{2}+i \varepsilon\left(\boldsymbol{l}_{j}^{2}+\mu_{j}^{2}\right) .
$$

* The research reported in this paper was supported in part by the National Science Foundation.

1 Commun.math. Phys., Vol.11 
The four vectors $\boldsymbol{l}_{j}$ are of the form

$$
l_{j}=\sum_{j^{\prime}=1}^{m} c_{j j^{\prime}} k_{j^{\prime}}+q_{j} .
$$

We further introduce the Euclidean integral corresponding to (2.1)

with

$$
I_{E}(q \mu)=\int d k \frac{P(k q)}{\prod_{j=1}^{n} e_{j}\left(k q_{j} \mu_{j}\right)}
$$

$$
e_{j}\left(k q_{j} \mu_{j}\right)=l_{j 0}^{2}+l_{j}^{2}+\mu_{j}^{2} .
$$

The following theorem shows that both integrals (2.1) and (2.5) have the same convergence properties.

Theorem 1. The Minkowskian integral (2.1) is absolutely convergent (a.c.) if and only if the corresponding Euclidean integral is a.c.

Proof. The inequalities

imply

$$
\begin{aligned}
& \frac{\boldsymbol{l}^{2}+\mu^{2}}{\left|l_{0}^{2}-\boldsymbol{l}^{2}-\mu^{2}+i \varepsilon\left(\boldsymbol{l}^{2}+\mu^{2}\right)\right|} \leqq \frac{1}{\varepsilon} \\
& \frac{l_{0}^{2}}{\left|l_{0}^{2}-\boldsymbol{l}^{2}-\mu^{2}+i \varepsilon\left(\boldsymbol{l}^{2}+\mu^{2}\right)\right|} \leqq \sqrt{1+\frac{1}{\varepsilon^{2}}}
\end{aligned}
$$

$$
\frac{l_{0}^{2}+l^{2}+\mu^{2}}{l_{0}^{2}-l^{2}-\mu^{2}+i \varepsilon\left(l^{2}+\mu^{2}\right)} \leqq \frac{1}{\varepsilon}+\sqrt{1+\frac{1}{\varepsilon^{2}}} .
$$

The Minkowskian integral (2.1) is therefore majorized by the Euclidean integral (2.5). Also (2.5) is majorized by (2.1) according to the inequality

$$
\frac{\left|l_{0}^{2}-\boldsymbol{l}^{2}-\mu^{2}+i \varepsilon\left(\boldsymbol{l}^{2}+\mu^{2}\right)\right|}{l_{0}^{2}+\boldsymbol{l}^{2}+\mu^{2}} \leqq \sqrt{1+\varepsilon^{2}}
$$

Combining Theorem 1 with Weinberg's version of the power counting theorem for Euclidean metric [1-3] one obtains

Theorem 2. Let all masses $\mu_{j} \neq 0$. The integral (2.1) is a.c. if (2.1) and any subintegral

$$
I(q \mu \varepsilon H)=\int_{H} d V \frac{P(k q)}{\prod_{j=1}^{n} f_{j}\left(k q_{j} \mu_{j} \varepsilon\right)}
$$

have negative dimension. $H$ denotes a hyperplane in $R_{4 m}$ described by a set of linear equations

$$
\sum_{j=1}^{m} d_{i j} k_{j}=r_{i}, \quad i=1, \ldots, t .
$$

The dimension of a rational integral is defined by $d=d^{\prime}+d^{\prime \prime}$ where $d^{\prime}$ is the number of integration variables and $d^{\prime \prime}$ the degree of the integrand with respect to the integration variables. 


\section{Parametrized Integrals}

Introducing Feynman parameters $\alpha=\left(\alpha_{1} \ldots \alpha_{n}\right)$ in the usual manner we obtain from (2.1)

with

$$
I(q \mu \varepsilon)=(n-1) ! \int d k \int_{\mathscr{D}} d \alpha \frac{P(k q)}{\left(\sum_{j=1}^{n} \alpha_{j} f_{j}\left(k q_{j} \mu_{j} \varepsilon\right)\right)^{n}}
$$

$$
d \alpha=d \alpha_{1} \ldots d \alpha_{n-1}, \alpha_{n}=1-\sum_{j=1}^{n-1} \alpha_{i}
$$

$\mathscr{D}$ is the set of all points $\left(\alpha_{1} \ldots \alpha_{n-1}\right)$ satisfying

$$
\alpha_{1} \geqq 0, \ldots, \alpha_{n} \geqq 0 \quad \text { and } \quad \sum_{i=1}^{n} \alpha_{i}=1 .
$$

For deriving the parametrized integral it is necessary to interchange the $k$ - and $\alpha$-integration in (3.1). To justify this we prove the following Lemma.

Lemma 1. If the k-integral (2.1) is a.c. the $k$ - $\alpha$-integral (3.1) is also a.c.

Proof. According to Theorem 1 the a.c. of (2.1) implies the convergence of

Hence also

$$
\bar{I}_{E}=\int d k \frac{|P(k q)|}{\prod_{j=1}^{n} e_{j}\left(k q_{j} \mu_{j}\right)} .
$$

is convergent. Since

$$
\bar{I}_{E}=(n-1) ! \int d k \int_{\mathscr{D}} d \alpha \frac{|P(k q)|}{\left(\sum_{j=1}^{n} \alpha_{j} e_{j}\left(k q_{j} \mu_{j}\right)\right)^{n}}
$$

the integral

$$
\frac{\sum_{j=1}^{n} \alpha_{j} e_{j}\left(k q_{j} \mu_{j}\right)}{\left|\sum_{j=1}^{n} \alpha_{j} f_{j}\left(k q \mu_{j} \varepsilon\right)\right|} \leqq \frac{n}{\varepsilon}+n \sqrt{1+\frac{1}{\varepsilon^{2}}}
$$

$$
\int d k \int_{\mathscr{D}} d \alpha \frac{|P(k q)|}{\left|\sum_{j=1}^{n} \alpha_{j} f_{j}\left(k q_{j} \mu_{j} \varepsilon\right)\right|^{n}}
$$

is majorized by (3.4). Hence (3.1) is a.c.

By FubinI's theorem it is therefore permitted to interchange the $k$ - and $\alpha$-integration in (3.1). We thus obtain

$$
\begin{aligned}
I(q \mu \varepsilon) & =\int_{\mathscr{D}} d \alpha B(q \mu \alpha \varepsilon)=\int_{\mathscr{D}-\mathscr{\delta}} d \alpha B(q \mu \alpha \varepsilon), \\
B(q \mu \alpha \varepsilon) & =(n-1) ! \int d k \frac{P(k q)}{\left(\sum_{j=1}^{n} \alpha_{j} f_{j}\left(k q_{j} \mu_{j} \varepsilon\right)\right)^{n}} .
\end{aligned}
$$

Here $\mathscr{E}$ denotes the set of all $\alpha \in \mathscr{D}$ for which at least one $\alpha_{i}=0$ $(i=1, \ldots, n)$. The integral $B$ can be evaluated by standard methods. 
We shortly indicate the derivation. Since

$$
\alpha_{1}>0, \ldots, \alpha_{n}>0 \text { in } \mathscr{D}-\mathscr{E}
$$

we need compute $B$ for positive $\alpha$ only. We consider the quadratic form

$$
F(k q \alpha)=\sum_{i=1}^{n} \alpha_{i} l_{i}^{2}=\sum a_{i j} k_{i} k_{j}+2 \sum Q_{j} k_{j}+Q .
$$

The matrix $a_{i j}$ depends on $\alpha$ only and has the properties

$$
\begin{aligned}
a_{i j} & =a_{j i} \\
d(\alpha) & =\operatorname{det}\left(a_{i j}\right)>0 \text { for } \alpha_{1}, \ldots, \alpha_{n}>0 .
\end{aligned}
$$

The stationary value of $F$ is

where $k^{0}$ is the solution of

$$
F_{0}(q \alpha)=F\left(k^{0} q \alpha\right)
$$

One obtains

$$
\sum_{j=1}^{m} a_{i j} k_{j}^{0}+Q_{i}=0
$$

$$
F=\sum a_{i j} k_{i}^{\prime} k_{j}^{\prime}+F_{0}(q \alpha)=\sum_{j=1}^{m} \lambda_{j} \tilde{k}_{j}^{2}+F_{0}(q \alpha)
$$

with the transformations

$$
k_{i}^{\prime}=k_{i}-k_{i}^{0}, \quad \tilde{k}_{i}=\sum_{j=1}^{m} O_{i j} k_{j}^{\prime}, \quad O=\left(O_{i j}\right) \text { orthogonal } .
$$

With this result we obtain for the denominator of (3.8)

$$
\begin{aligned}
\sum_{j=1}^{n} \alpha_{j} f_{j}\left(k q_{j} \mu_{j} \varepsilon\right) & =\sum_{j=1}^{m} \lambda_{j} \tilde{k}_{j}^{2}+i \varepsilon \sum_{j=1}^{m} \lambda_{j} \tilde{\boldsymbol{k}}_{j}^{2}+C, \\
C & =F_{0}(q \alpha)+i \varepsilon F_{0}(\boldsymbol{q} \alpha)-(1-i \varepsilon) \sum_{j=1}^{n} \alpha_{j} \mu_{j}^{2}, \\
F_{0}(\boldsymbol{q} \alpha) & =F_{0}(q \alpha) \quad \text { with } \quad q_{j}^{0}=0 .
\end{aligned}
$$

Introducing the polynomial $\bar{P}$ by

we obtain

$$
\begin{aligned}
\bar{P}(\bar{k} q \alpha) & =P(k q) \\
k & =O^{-1} \lambda^{-1 / 2} \bar{k}+k^{0}
\end{aligned} \quad \lambda=\left(\lambda_{i} \delta_{i j}\right)
$$

$$
B=\frac{(n-1) !}{d(\alpha)^{2}} \int d k \frac{\bar{P}(k q \alpha)}{\left(\sum k_{j}^{2}+i \varepsilon \sum \boldsymbol{k}_{j}^{2}+C\right)^{n}} .
$$

We decompose $\bar{P}$ into parts which are homogeneous in each $k_{i \mu}$ separately

So $B$ becomes

$$
\bar{P}(k q \alpha)=\sum_{v_{10} \ldots v_{m 3}} \prod_{i=0}^{m} \prod_{\mu=0}^{3}\left(k_{i \mu}\right)^{v_{i \mu}} \prod_{v_{10} \ldots v_{m 3}}(q \alpha) .
$$

$$
B=\sum_{\gamma_{10} \ldots \gamma_{m 3}} B_{\gamma_{10} \ldots \gamma_{m 3}} \Pi_{2 \gamma_{10}, \ldots, 2 \gamma_{m 3}}
$$


with the coefficients

$$
B_{\gamma_{10} \ldots \gamma_{m 3}}=\frac{(n-1) !}{d(\alpha)^{2}} \int d k \frac{\left(k_{10}\right)^{2} \gamma_{10} \ldots\left(k m_{3}\right)^{2 \gamma_{m 3}}}{\left(\sum k_{j}^{2}+i \varepsilon \sum \boldsymbol{k}_{j}^{2}+C\right)^{n}} .
$$

The remaining integrations are elementary and can easily be carried out. We state the final result in the following lemma.

Lemma 2. If the integral (2.1) is a.c. it can be brought into the parametrized form

$$
I(q \mu \varepsilon)=\int_{\mathscr{D}} d \propto B(q \mu \alpha \varepsilon)
$$

where (except for the set $\mathscr{E}$ of measure zero) the integrand $B$ is given by

$$
B(q \mu \propto \varepsilon)=\sum_{\gamma_{10} \ldots \gamma_{m 3}} B_{\gamma_{10} \ldots \gamma_{m 3}}(q \propto \varepsilon) \Pi_{2 \gamma_{10}, \ldots, 2 \gamma_{m 3}}(q \alpha) .
$$

The $I I$ are polynomials in $q$ defined by (3.18). The functions $B$ are explicitly

with

$$
B_{\gamma_{10} \ldots \gamma_{m 3}}=\frac{\left(\gamma_{10}\right) \ldots\left(\gamma_{m 3}\right)(n-\gamma-2 m-1) ! \pi^{2} i^{m}}{2^{\gamma}(i \varepsilon-1)^{\sigma} d(\alpha)^{2} C^{n-\gamma-2 m}}
$$

$$
\begin{aligned}
C & =F_{0}(q \alpha)+i \varepsilon F_{0}(\boldsymbol{q} \alpha)-(1-i \varepsilon) \sum_{j=1}^{n} \alpha_{j} \mu_{j}^{2}, \\
\gamma & =\sum_{j=1}^{n} \sum_{\mu=0}^{3} \gamma_{j \mu}, \\
\sigma & =\sum_{j=1}^{n} \sum_{\mu=1}^{3} \gamma_{j \mu}+\frac{3 m}{2}, \\
(N) & =1 \cdot 3 \cdot 5 \ldots(2 N-1) \text { for } N=1,2, \ldots, \\
(0) & =1 .
\end{aligned}
$$

Formula (3.23) is still unsatisfactory insofar as the denominator contains terms which are not covariant. The following theorem, however, shows that these terms may be dropped in the limit $\varepsilon \rightarrow+0$. Since this limit will be considered in the sense of distributions we have to specify the domain of definition for the variables $q_{1} \ldots q_{n}$. We assume that $q$ varies over a $4 r$-dimensional vector space described by

$$
\begin{aligned}
& q=\beta p \\
& \beta=\left(\beta_{i j}\right), \quad p=\left(p_{1} \ldots p_{r}\right), \quad r \leqq n
\end{aligned}
$$

where the $p_{j}$ are arbitrary four vectors and the $n \times r$ matrix $\beta$ has rank $r$. This assumption covers all situations where Feynman integrals are considered as distributions in the external variables.

Theorem 3. For $\varepsilon \rightarrow+0$ the functions $I(q(p), \mu, \varepsilon)$ converge strongly in $\mathscr{S}^{\prime}\left(R_{4 r}\right)$ to a tempered distribution

$$
I(q(p), \mu)=\lim _{\varepsilon \rightarrow+0} I(q(p), \mu, \varepsilon) \text { in } \mathscr{S}^{\prime}\left(R_{4 r}\right) .
$$


$I(q(p), \mu)$ can as well be expressed as the strong limit of covariant parametrized integrals

where

$$
I(q(p), \mu)=\lim _{\varepsilon \rightarrow+0} \Phi(q(p), \mu, \varepsilon) \text { in } \mathscr{S}^{\prime}\left(R_{4 r}\right)
$$

$$
\begin{aligned}
\Phi(q \mu \varepsilon) & =\int_{\mathscr{D}} d \alpha \Psi(q \mu \alpha \varepsilon) \\
\Psi(q \mu \alpha \varepsilon) & =\sum_{\gamma_{10} \ldots \gamma_{m 3}} \Xi_{\gamma_{10} \ldots \gamma_{m 3}}(q \alpha) \Pi_{2 \gamma_{10}, \ldots, 2 \gamma_{m 3}}(q \alpha) \\
\Xi_{\gamma_{10} \ldots \gamma_{m 3}} & =\frac{\left(\gamma_{10}\right) \ldots\left(\gamma_{m 3}\right)(n-\gamma-2 m-1) ! \pi^{2} i^{m}}{2^{\gamma} d(\alpha)^{2} \Delta^{n-\gamma-2 m}} \\
\Delta & =F_{0}(q \alpha)-\sum_{j=1}^{m} \alpha_{j} \mu_{j}^{2}+i \varepsilon \sum_{j=1}^{m} \alpha_{j} \mu_{j}^{2} .
\end{aligned}
$$

The distribution $I(q(p), \mu)$ is explicitly given by the mapping

$$
\varphi(p) \rightarrow \lim _{\varepsilon \rightarrow+0} \int d p \varphi(p) \Phi(q(p), \mu, \varepsilon) \text { for } \varphi \in \mathscr{S}\left(R_{4 r}\right) .
$$

Proof. The limit $\varepsilon \rightarrow+0$ of parametrized Feynman integrals has been discussed by HEPP [4]. Since the integral (3.21-23) is of a slightly different type we first reformulate the problem such that Hepp's method can be applied.

The function $I(q \mu \varepsilon)$ is of the form

$$
\begin{aligned}
I(q(p), \mu, \varepsilon) & =\int_{\mathscr{D}} B(q(p), \mu, \alpha, \varepsilon) \\
B(q(p), \mu, \alpha, \varepsilon) & =\frac{N(p \mu \varepsilon \alpha)}{(i \varepsilon-1)^{s} C^{n-2 m}}
\end{aligned}
$$

where $N$ is a polynomial in $p$ and $\varepsilon$. The function $I(q(p), \mu, \varepsilon)$ converges in $\mathscr{S}^{\prime}\left(R_{4 r}\right)$ to a distribution if the limit

$$
\lim _{\varepsilon \rightarrow+0} \int d p \varphi(p) I(q(p), \mu, \varepsilon)
$$

exists for every $\varphi \in \mathscr{S}\left(R_{4 r}\right)$. Hence the existence of (3.31) must be shown.

We have

$$
\begin{gathered}
\int d p \varphi(p) I(q(p), \mu, \varepsilon) \\
=\sum_{v_{10} \ldots v_{r} \nu} \int d p \int_{\mathscr{D}} d \alpha \varphi(p) N_{v_{10} \ldots v_{r} 3}^{(\nu)}(\mu \alpha) \frac{\varepsilon^{\nu} P_{10}^{v_{10}} \ldots P_{r 3}^{v_{r} 3}}{(i \varepsilon-1)^{s} C^{n-2 m}}
\end{gathered}
$$

where $N_{v_{10} \ldots v_{r} 3}^{(v)}$ denote the coefficients of the polynomial $N$ with respect to $p$ and $\varepsilon$

$$
N(q(p), \mu, \alpha, \varepsilon)=\sum_{v_{10} \ldots v_{r 3} \nu} N_{v_{10} \ldots v_{r} 3}^{(\nu)}(\mu \alpha) \varepsilon^{v} p_{10}^{v_{10}} \ldots p_{r 3}^{v_{r} 3} .
$$

Each term on the right hand side of (3.32) is a.c. with respect to the $p$ - and $\alpha$-integration as can be seen in the following way. First we note that (3.30) is a.c. since

$$
\int d \alpha|B(q \mu \alpha \varepsilon)| \leqq(n-1) ! \int_{\mathscr{D}} d \alpha \int d k\left|\frac{P(k q)}{\left(\sum \alpha_{j} f_{j}\left(k q_{j} \mu_{j}\right)\right)^{n}}\right|
$$


(Lemma 1, p. 3). The a.c. of (3.30) implies the a.c. of

$$
\int_{\mathscr{D}} d \propto N(q \mu \alpha \varepsilon)
$$

because

$$
|C| \leqq M \text { for } \alpha \in \mathscr{D} .
$$

Applying Lemma 3 a of ref. [3] we obtain that

$$
\int_{\mathscr{D}} d \alpha N_{v_{10} \ldots v_{r} 3}^{(\nu)}(\mu \alpha) \text { is a.c. }
$$

On the other hand we have $|C| \geqq \gamma>0$ for $\alpha \in \mathscr{D}$ since $\mu_{i}>0$. Hence each term on the right hand side of (3.32) is a.c. according to

$$
\begin{gathered}
\int d p \int_{\mathscr{D}} d \alpha\left|\varphi(p) N_{v_{10} \ldots v_{r} 3}^{(\nu)} \frac{p_{10}^{\nu_{10}} \ldots p_{r 3}^{\nu_{r} 3}}{C^{n-2 m}}\right| \\
\leqq A \int d p\left|\varphi(p) p_{10}^{\nu_{10}} \ldots p_{r 3}^{v_{r} 3}\right| \int_{\mathscr{D}} d \alpha\left|N_{v_{10} \ldots v_{r}}^{(\nu)}\right| .
\end{gathered}
$$

It is therefore permitted to interchange the $p$ - and $\alpha$-integration in (3.32) and we obtain

$$
\begin{gathered}
\int d p \varphi(p) I(p \mu \varepsilon) \\
=\sum_{v_{10} \ldots v_{r} 3^{v}} \int_{\mathscr{D}} d \alpha N_{v_{10} \ldots v_{r} 3}^{(v)}(\mu \alpha) \int d p \frac{\varepsilon^{v} p_{10}^{v_{10}} \ldots p_{r 3}^{v_{r} 3} \varphi(p)}{(i \varepsilon-1)^{s} C^{n-2 m}} .
\end{gathered}
$$

For proving the existence of (3.31) it is therefore sufficient to show that the limit $\varepsilon \rightarrow+0$ of

$$
\int_{\mathscr{D}} d \propto \int d p \psi(p) \frac{N_{v_{10} \ldots \nu_{r 3}(\mu \alpha)}^{(\nu)}}{C^{n-2 m}}
$$

exists for every $\psi \in \mathscr{S}\left(R_{4 r}\right) . C$ is given by (3.24) with $q(p)$ substituted for $q . F_{0}(q(p), \alpha)$ is a quadratic form in the $p_{j}$

$$
F_{0}(q(p), \alpha)=\sum_{i, j=1}^{r} A_{i j}(\alpha) p_{i} p_{j}
$$

(3.37) has the form of the parametrized integrals for which the limit $\varepsilon \rightarrow+0$ was studied by HePP (Eq. (4.9-10) of ref. [4]). According to (3.35) the coefficients $N_{v_{10} \ldots v_{r} 3}^{(v)}$ are absolutely integrable in $\mathscr{D}$. We will further show that the coefficients $A_{i j}(\alpha)$ (originally defined for $\alpha_{j}>0$ ) can be extended to continuous functions of $\alpha$ in $\alpha_{j} \geqq 0$. To this end we introduce the quadratic form

$$
\begin{aligned}
G(x y \alpha) & =\sum_{i=1}^{n} \alpha_{i} z_{i}^{2} \\
z_{i} & =\sum_{j=1}^{n} c_{i j} x_{j}+\sum_{j=1}^{r} \beta_{i j} u_{j}
\end{aligned}
$$


which is obtained from $F(k, q(p), \alpha)$ by replacing the four vectors $k_{j}, p_{j}$ by real variables $x_{j}$ or $y_{j}$ resp. Then we define

$$
G_{0}(y \alpha)=\operatorname{Inf}_{x} G(x y \alpha)=\sum_{i, j=1} A_{i j}(\alpha) y_{i} y_{j} .
$$

Here the $A_{i j}(\alpha)$ are continuous functions of $\alpha$ in $\alpha_{i} \geqq 0$ and coincide with the coefficients of (3.38) for $\alpha_{j}>0$.

Following HEPP it can now be shown that the limit (3.31) exists. Hence $I(q(p), \mu, \varepsilon)$ approaches a distribution in $\mathscr{S}^{\prime}\left(R_{4 r}\right)$ for $\varepsilon \rightarrow+0$.

By a similar argument it follows that also $\Phi(q(p), \mu, \varepsilon)$ converges in $\mathscr{S}^{\prime}\left(R_{4 r}\right)$ for $\varepsilon \rightarrow+0$.

We further have

since

$$
\lim _{\varepsilon \rightarrow+0}(I(q(p), \mu, \varepsilon)-\Phi(q(p), \mu, \varepsilon))=0
$$

$$
\lim _{\varepsilon \rightarrow+0} \int d q \varphi(q)\left\{\frac{1}{(1-i \varepsilon)^{\sigma} C^{n-2 m-\gamma}}-\frac{1}{\Delta^{n-2 m-\gamma}}\right\}=0
$$

for every $\varphi \in \mathscr{S}\left(R_{4 r}\right)$.

This completes the proof of the theorem.

\section{References}

1. Dyson, F. J.: Phys. Rev. 75, 1736 (1949).

2. Weinberg, S.: Phys. Rev. 118, 838 (1960).

3. Hahn, Y., and W. ZimmermanN: To be published.

4. Hepr, K.: Commun. Math. Phys. 2, 301 (1966).

W. ZimmermanN

Courant Institute of Math. Sciences

251 Mercer Street

New York, N. Y. 10012, USA 